Relations industrielles

Industrial Relations

\title{
Conflits et résistances au travail, Par Yvan Sainsaulieu (2017) \\ Paris : Presses de Science Po, 180 pages. ISBN : 978-2-7246-2080-1
}

\section{Christian Thuderoz}

Volume 73, numéro 1, hiver 2018

URI : https://id.erudit.org/iderudit/1044433ar

DOI : https://doi.org/10.7202/1044433ar

Aller au sommaire du numéro

Éditeur(s)

Département des relations industrielles de l’Université Laval

ISSN

0034-379X (imprimé)

1703-8138 (numérique)

Découvrir la revue

Citer ce compte rendu

Thuderoz, C. (2018). Compte rendu de [Conflits et résistances au travail, Par

Yvan Sainsaulieu (2017) Paris : Presses de Science Po, 180 pages. ISBN :

978-2-7246-2080-1]. Relations industrielles / Industrial Relations, 73(1), 207-209.

https://doi.org/10.7202/1044433ar

Tous droits réservés @ C Département des relations industrielles de l’Université Laval, 2018
Ce document est protégé par la loi sur le droit d'auteur. L'utilisation des services d’Érudit (y compris la reproduction) est assujettie à sa politique d'utilisation que vous pouvez consulter en ligne.

https://apropos.erudit.org/fr/usagers/politique-dutilisation/ 
globalization and the changing nature of professional work, discourse and identity amongst professional workers. I also found some contributions on professions seldom written about (architects, for example) especially interesting. On the other hand, some large professions such as engineers barely rate a mention. While selectivity is inevitable with such a diverse array of vocations that might be labelled as professionals, the editors could have spent more time trying to place their work into a wider context. Indeed, this is one of the major limitations of the book. The editorial introduction chapter spends only four pages describing the overall thrust of the book and key themes, with the remainder of the chapter providing roughly half-page summaries of each of the contributing chapters. To be fair, these descriptions do point to key themes, but the reader is largely left to tease the unifying or overlapping themes for themselves-something the index only partly makes up for. In my view, a more synthesized introduction or final chapter was needed and would really have enhanced the value of the book.

My other quibble, which may flow on from the last, is that the book suffers from two glaring and probably related omissions, namely there is little reference to the regulation/certification/licensing of professions and the historical context of this, especially debates over public safety. This is a book locked very much into contemporary discourse including identity analysis, if one looks at the only explicitly historical chapter, which deals with how a family moved into the professions over time. The latter was interesting but hardly a substitute for a historical analysis of the critical role played by public safety concerns in regulating certain professions requiring technical expertise before completing tasks (mine managers, shipmasters, airline pilots, engineers, medical practitioners to name but a few). Economists portray this as rent seeking and I would like to suggest they test their convictions by opting to take a cheaper flight in a plane with an unlicensed pilot and maintained by unlicensed aircraft engineers and being treated by unlicensed medical professionals if they survive a crash. The point is that licensing professions occurred for a reason, and after long campaigns (see, for example, Turner, 1989), although now under attack from economists and those who argue safety systems can safeguard people. In short, the history of regulation of professions is important; not only to understanding the past, but also in helping us take a more critical view of contemporary debates. The editors would have assisted readers, I think, by covering this terrain even if briefly.

In a sense, notwithstanding their value, both books share a flaw in that in the current discourse on work history and regulation do not seem to matter as much as, in my view, they should.

\section{Michael Quinlan \\ Professor \\ School of Management \\ UNSW Business School, Australia}

\section{References}

Fry, S. and B. Mees (2017) "Two Discursive Frameworks Concerning Ideology in Australian Industrial Relations", Economic and Labour Relations Review, 28 (4), 483-499.

Turner, K. (1989) "Safety, Discipline and the Manager: Building a 'Higher Class of Men'", Sociology, 23 (4), 611-628.

\section{Conflits et résistances au travail}

Par Yvan Sainsaulieu (2017) Paris : Presses de Science Po, 180 pages.

ISBN : 978-2-7246-2080-1.

Publié dans la collection "Contester» des Presses de Sciences Po, ce treizième opus - qui fait suite à des ouvrages comme $L a$ Grève (de Guy Groux et Jean-Marie Pernot, 2008), La Manifestation (d'Olivier Filleule et Danièle Tartakowsky, 2008), La Désobéissance civile (de Grame Hayes et Sylvie Ollitrault, 2012) ou encore Le Boycott ( $d^{\prime}$ Ingrid 
Nyström et Patricia Vendramin, 2015) cet opus, donc, constitue une bonne synthèse du savoir académique contemporain sur le conflit au travail (et pas seulement : le conflit $d u$ travail). Le format de cette collection - 155 pages de texte, sans compter la bibliographie et les tables des matières - invite à cette nécessaire concision, sans écorner le fond du propos : comprendre la contestation au travail dans la diversité et la richesse de ses expressions.

L'ouvrage n'est pas seulement une synthèse des travaux sur le sujet; il propose également une lecture singulière du phénomène. L'auteur, fils d'un sociologue de l'entreprise et des relations sociales, Renaud Sainsaulieu, dont les travaux, originaux, marquèrent une génération de chercheurs et d'enseignants, a su, en effet, rendre compte de la pluralité des situations de conflit et de résistance au travail, sans les subsumer dans une seule de leurs formes, érigée en matrice dont tout découlerait le conflit du travail "classique», la grève, donc, arme archétypique du mouvement ouvrier et expression vivante de son refus de la domination de classe - et fournir les éléments de compréhension de cette conflictualité, alors que changent les mondes du travail, le travail lui-même, et la manière dont sont désormais gouvernés les hommes et les femmes au travail.

Pluralité des situations, d'abord, décrite et analysée au cours des quatre premiers chapitres qui composent l'ouvrage : celle de la grève, avec son répertoire d'actions collectives et ses animateurs syndicaux; celle du conflit postindustriel ou tertiaire; celle des conflits identitaires et professionnels; enfin, celle de la discorde au travail.

Le conflit au travail, note l'auteur en liminaire du premier chapitre, intitulé «Grèves et action syndicale», "appartient d'abord à I'histoire du mouvement ouvrier» (p. 25); comprendre cette conflictualité, y compris dans son déclin contemporain, c'est donc interroger le syndicalisme, dont il en a fait I'arme collective par excellence. Le deuxième chapitre, «Du conflit post-industriel et tertiaire », décrit (trop sommairement, cependant) les situations de résistance dans divers lieux, de I'hôpital au supermarché, ou nouées par divers acteurs, des femmes de ménage aux intermittents du spectacle. Le troisième chapitre, "Professionnels et cadres en résistance», porte le regard sur une action contestataire plus diffuse, celle des travailleurs hautement qualifiés, entre souffrance et frustration.

Reste la «discorde au travail». Sous cette expression, titre du quatrième chapitre, Sainsaulieu regroupe «les conflits de personnes, affectifs, culturels, d'opinions, de pouvoirs ou d'intérêts», dont la particularité est de ne pas prendre pour cible «l'autorité supérieure». II explore ainsi ces "antagonismes plus horizontaux que verticaux», souvent tus ou négligés, car ne s'inscrivant pas "dans la contestation sociale des pouvoirs établis". S'ensuivent quelques pages, un peu touffues, mais qui ont le mérite de dissocier la conflictualité locale de "l'oppression macro-sociale», comme si celle-ci était la matrice originelle par laquelle tout s'expliquerait.

Des éléments de compréhension, ensuite, distillés tout au long de l'ouvrage. Les moindres ne sont pas ceux proposés dans le chapitre final, "Ancrage politique au travail» où l'auteur livre d'intéressantes (mais trop rapides) notations sur "l'insertion dans un milieu professionnel comme une expérience ordinaire susceptible d'avoir un impact politique» (p. 141). Même si les travaux d'Isabelle Ferreras ne sont pas ici mobilisés par l'auteur (elle est citée, ailleurs au détour d'une phrase sur «le sens moral attaché au travail », p. 68), ce dernier mentionne, avec raison, ces «rapports ordinaires au politique », peu étudiés à ce jour, et que produisent continument les milieux et les relations de travail.

Cet effort compréhensif se double d'un regard lucide sur quelques impensés de la sociologie des conflits sociaux. Sainsaulieu remarque ainsi, à juste titre, que «le 
débat sur l'articulation des organisations au mouvement social reste inabouti» (p. 46). Autrement dit : peu d'observations et peu de travaux publiés sur le rôle des syndicats dans le déclenchement, la poursuite et le règlement des conflits du travail. L'auteur y consacre quelques paragraphes, parfois elliptiques; ils ont le mérite de rappeler quelques vérités : "Les grèves participent ainsi de la dynamique scissionniste au sein du syndicalisme français» (p. 48) ; "Le climat social s'invite dans l'échange de coups entre militants» (p. 48).

Parfois, au détour d'une phrase, l'auteur laisse aller sa plume: "Les ruines fumantes de la capacité conflictuelle des salariés», lit-on curieusement page 44 ; ou plus loin : " C'est d'écrasement dont il est question» (p. 103). Ailleurs, il prend acte, avec raison, de la thèse de "l'indétermination managériale», qu'il estime «tout aussi défendable» que celle, inverse, de «la toute puissance managériale». Ou, quelques pages plus loin, il reconnaît que l'école du labour process (procès de travail) ne manque pas d'arguments pour affirmer qu'en pratique, sur le shopfloor (plancher de I'atelier), «domination et coopération sont étroitement mêlées» (p. 107). Ainsi va I'ouvrage, oscillant entre une lecture sociologique ouverte du conflit, tel que Georg Simmel l'a inauguré il y a déjà plus d'un siècle, et une lecture plus classique (et probablement dépassée) de ce même conflit, référé à une "conscience de classe» et ramené à son expression archétypique (la grève syndicalement encadrée).

D'où l'impression, pour le lecteur, d'un entre-deux permanent, comme si l'auteur n'osait franchir le pas et traiter de la conflictualité au travail sans devoir mobiliser les horizons lointains de la «mondialisation» ou de "la transformation globale des classes et du salariat». Car ce même lecteur, vivement intéressé par l'ambition initiale - ne pas «ignorer les logiques sectorielles ou locales au profit des seules logiques transversales» (p. 21); «ne pas raisonner dans un cadre unique »; et "faire dialoguer des perspectives différentes qui permettent de constituer une mosaïque riche de l'opposition au travail » (p. 22) - , et également acquis à l'idée « de logiques partielles qui peuvent se jouer et se rejouer à des échelles différentes » ( $p$. 153), est surpris de découvrir une question qu'il croyait dépassée et résolue ( «La logique de classe sort-elle invalidée de notre examen ?», p. 153). Pourquoi s'embarrasser ainsi, alors que l'ouvrage, ce qui fait son intérêt et sa nécessité, est construit sur une toute autre idée, celle d'un entrelacs de composantes sectorielles, organisationnelles et politiques de la conflictualité au travail, celle de l'interdépendance étroite entre travail et hors-travail, celle du rôle majeur des différences de genre et des discriminations ?

L'opposition au travail, dans les univers du travail contemporain, est diverse; I'ouvrage le montre avec éloquence, exemples et encadrés à l'appui. Et l'auteur a raison de distinguer «résistance au travail » — plutôt " un état d'esprit», dit-il, entre patience et endurance - et «conflit», celui-ci pouvant "s'étendre ou s'approfondir», "évoluer vers une forme plus contestataire " — ou, pensons-nous, se maintenir dans un cadre strictement local et organisationnel, ce qui est son régime ordinaire. Étudier le conflit au travail aujourd'hui, c'est donc se doter de grilles de lectures renouvelées, et Yvan Sainsaulieu y parvient fort bien; mais c'est aussi, sans tergiverser, se débarrasser des lunettes inappropriées. Certains types d'explication, notait Wittgenstein, exercent sur nous une attraction irrésistible; et celle-ci est plus grande que ce que nous pouvons concevoir de différent. Le moment est peutêtre venu de concevoir autrement le conflit au travail; Yvan Sainsaulieu propose ici de premières pistes; il convient, résolument, de prolonger l'effort.

\section{Christian Thuderoz}

Directeur du programme NégoLab

Essec-Irené, Paris 